\title{
Glacial acetic acid as an efficient catalyst for simple synthesis of dindolylmethanes
}

\author{
Mardia El-Sayed $^{\mathrm{a}, \mathrm{b}^{* *}}$, Kazem Mahmoud ${ }^{\mathrm{a}}$ and Andreas Hilgeroth ${ }^{\mathrm{a}}$
}

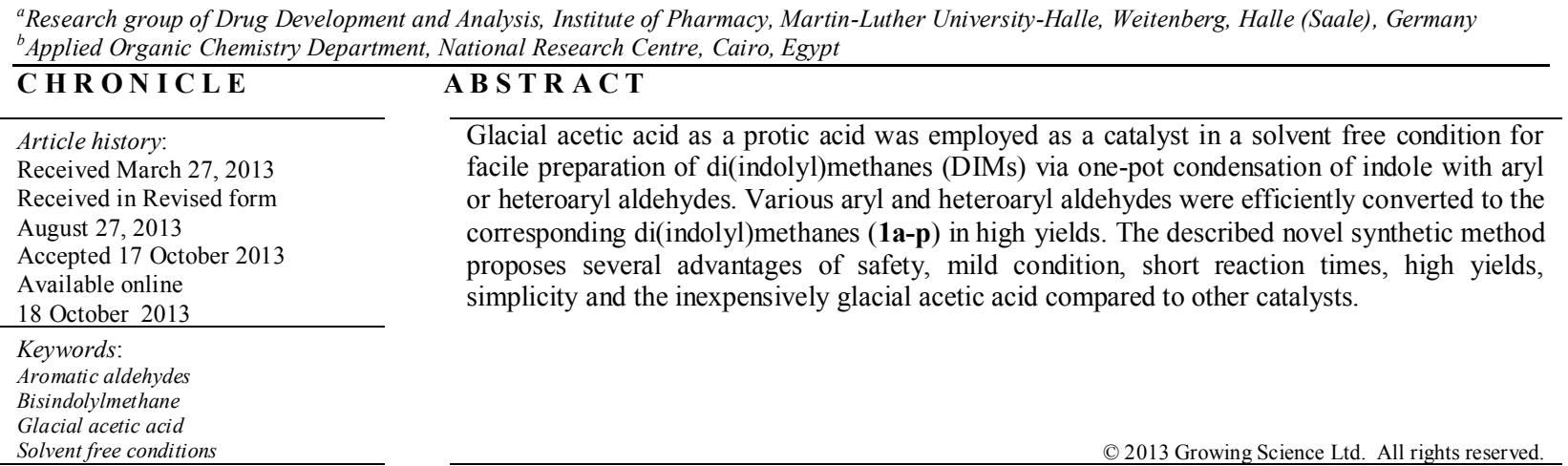

\section{Introduction}

Di(indolyl)methanes (DIMs) are molecules containing two indolyl moieties connected to the same carbon. Many advances in the strategy of DIMs synthesis were published as result of the variation of the catalyst. Other factors that prompted new research include the price of catalysts, yield of products, reaction rates, simplicity of the work up procedure, green chemistry, etc ${ }^{1 \mathrm{a}}$. Di(indolyl)alkanes and their derivatives are found in bioactive metabolites of terrestrial and marine origin ${ }^{1 \mathrm{~b}}$. A recent patent describes the synthesis of DIMs forming complex compounds with radioactive metal ions $\left(\mathrm{Gd}^{3+}\right)$, which are found to be useful contrast agents for radio-imaging and visualization of various tissues and organs ${ }^{2}$. Recently, Maciejewska et $\mathrm{al}^{3}$ used DNA-based electrochemical biosensors to demonstrate that bis(5-methoxyindol-3-yl)methane, considerably reduces the growth of the cancer cell lines such as HOP-92 (lung), A498 (renal) and MDAMB-231/1TCC (breast). Their results also indicated that BIMs could potentially be applied as chemotherapeutic agents against tumors ${ }^{1,3}$. BIMs and tris(indolyl)methanes (TIMs), have been used as legands for the synthesis of many complex 
molecules and different properties of these complex molecules have been investigated ${ }^{4-9}$. The electron rich indole nucleus shows an enhanced reactivity towards carbon electrophiles that generally results in the formation of three substituted indole derivatives ${ }^{10}$. The 3 -position of the indole is the preferred site for the electrophilic substitution reactions. 3-Alkyl or 3-acyl indoles are versatile intermediates for the synthesis of a wide range of indole derivatives ${ }^{11}$. A simple and direct method for the synthesis of 3-alkylated indoles involves the condensation with aliphatic or aromatic aldehydes.

Normally these reactions occur in presence of several types of catalysts for example protic or Lewis acids or ionic liquids ${ }^{12-39}$. As seen from these reported literature numerous catalysts can promote the reaction of aldehydes or ketones and indoles to afford 3-alkylated indole compounds in good to high yields in a reasonable time.

\section{Result and Discussion}

In the present work we wish to introduce $\mathrm{AcOH}$ as a mild and efficient catalyst for the promotion of the condensation reaction of indoles with aromatic aldehydes. In this paper we estimate the yield of BIMs via using glacial acetic acid in a solvent free condition with indoles and aromatic aldehydes. Some of these BIMs have been reported by using different types of catalysts as shown above. It has been found that, glacial acetic acid acts as a protic acid without solvent to catalyze the reaction of indoles (two equivalent moles) and aryl or heteroaryl aldehydes (one equivalent mole). Acetic acid has only been reported as a catalyst in the preparation of BIMs derived from 4-cyanoindole and formaldehyde solution. The reaction was done by using drops of acetic acid and finished after about $60 \mathrm{~h}^{23,24}$. In our present work via glacial acetic acid as a solvent (5 ml) the corresponding BIMs were given in high yields $(73-98 \%)$ and after few hours $(4-6 \mathrm{~h})$ of stirring at room temperature. In comparison to the reported methods, glacial acetic acid under a solvent free condition was found to be an efficient catalyst in terms of handling, temperature, yields and reaction times, (Scheme 1 and Table 1).

Table 1. Synthesized BIMs

\begin{tabular}{|c|c|c|c|c|c|}
\hline Entry & Aldehydes & Indoles & Product & Reaction time (h) & Yield (\%) \\
\hline 1 & $\mathrm{R}=\mathrm{Ph}$ & Indole & $1 \mathrm{a}$ & 5 & 90 \\
\hline 2 & $\mathrm{R}=p-\mathrm{NO} 2-\mathrm{Ph}$ & “ & $1 b$ & 4 & 98 \\
\hline 3 & $\mathrm{R}=p-\mathrm{Br}-\mathrm{Ph}$ & “" & 1c & 6 & 99 \\
\hline 4 & $\mathrm{R}=p-\mathrm{Cl}-\mathrm{Ph}$ & “ & 1d & 5 & 76 \\
\hline 5 & $\mathrm{R}=p-\mathrm{N}(\mathrm{Me}) 2-\mathrm{Ph}$ & “ & 1e & 5 & 91 \\
\hline 6 & $\mathrm{R}=m-\mathrm{Br}-\mathrm{Ph}$ & “" & 1f & 4 & 88 \\
\hline 7 & $\mathrm{R}=m-\mathrm{OCH}_{2} \mathrm{Ph}-\mathrm{Ph}$ & “ & $1 g$ & 5 & 87 \\
\hline 8 & $\mathrm{R}=p, m-\mathrm{OH}-\mathrm{Ph}$ & “ & $1 \mathrm{~h}$ & 6 & 73 \\
\hline 9 & $\mathrm{R}=p-\mathrm{MeO}-m-\mathrm{OCH}_{2} \mathrm{Ph}-\mathrm{Ph}$ & “ & $\mathbf{1 i}$ & 4 & 89 \\
\hline 10 & $\mathrm{R}=m-\mathrm{MeO}-p-\mathrm{OCH}_{2} \mathrm{Ph}-\mathrm{Ph}$ & “" & $\mathbf{1 j}$ & 5 & 92 \\
\hline 11 & $\mathrm{R}=m-\mathrm{Me}, 2,4,6$-tri-F-Ph & “ & $1 \mathbf{k}$ & 6 & 77 \\
\hline 12 & $\mathrm{R}=1$-naphthyl & “" & 11 & 4 & 97 \\
\hline 13 & $\mathrm{R}=3$-pridyl & “" & $1 \mathrm{~m}$ & 6 & 95 \\
\hline 14 & $\mathrm{R}=3$-indolyl & “" & 1n & 6 & 98 \\
\hline 15 & $\mathrm{R}=p-\mathrm{MeO}-m-\mathrm{OCH}_{2} \mathrm{Ph}-\mathrm{Ph}$ & 5-Cl-indole & 10 & 4 & 91 \\
\hline 16 & $\mathrm{R}=p-\mathrm{MeO}-m-\mathrm{OCH}_{2} \mathrm{Ph}-\mathrm{Ph}$ & 6-Cl-indole & $1 p$ & 4 & 93 \\
\hline
\end{tabular}

A series of substituted aryl or heteroaryl aldehydes were efficiently converted to the corresponding BIMs 1a-p, as shown in Table 1, which give the reaction times and the formed yields. Concerning the substituent on the carbonyl compounds, we can summarize that the presence of either electron donating group (such as dimethylamino, methoxy, benzyloxy or hydroxy) or electron with-drawing group (e.g. nitro, chloro, bromo or trifluoro) has not noticeable effect on the reaction time or the percent of the yield. So we can conclude that glacial acetic acid promotes the electrophilic substitution reaction of indoles with aromatic aldehydes whatever the substituent on the aromatic aldehyde and this makes it different from all the catalysts used in these reactions. In addition the substituent on the indole phenyl ring (5-chloro and 6-chloro indole) plays a role in 
the reaction which partially enhances the product formation as indicated by the same reaction time and higher yield (entry 15 and 16). BIMs 1a-f, and 1l-n are known ${ }^{12-39}$ and their identities were proven by means of MS, NMR, and IR spectra, and the other BIMs (1g-k, 1o-p), are novel and could not be found in the literature.
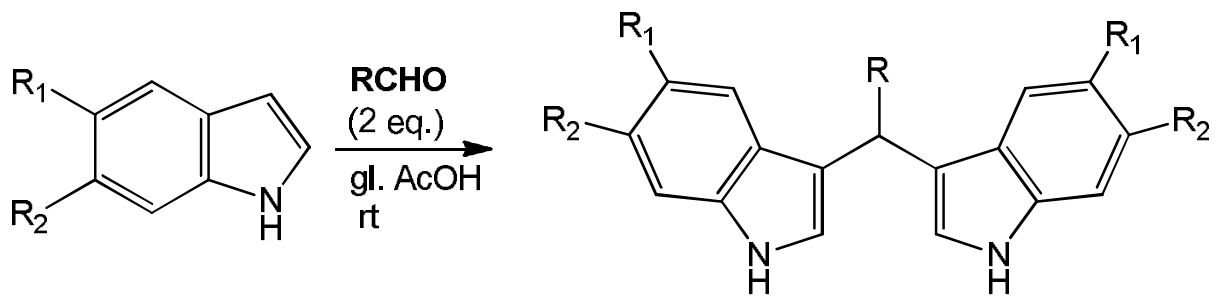

1a-p

Scheme 1. Synthesis of BIMs.

\section{Conclusion}

Glacial acetic acid was used as a catalyst and solvent for a facile synthesis of di(indolyl)methanes (DIMs) with high yields via one-pot condensation of indole with aryl or heteroaryl aldehydes. The described novel synthesis method proposes several advantages of safety, mild condition, short reaction times, high yields, simplicity and the inexpensively glacial acetic acid compared to other catalysts.

\section{Acknowledgments}

The authors are grateful to Egyptian culture affairs and missions sectors for the financial support as $\mathrm{PhD}$ scholarship for Mardia El-Sayed at the Martin Luther university-Halle Wittenberg, Germany.

\section{Experimental}

\subsection{Materials and Methods}

The melting points were measured on a Boetius-Mikroheiztisch the company "VEB weighing. Rapido Radebeul / VEB NAGEMA "measured and are uncorrected. TLC for the analyzes were with aluminium foil fluorescent indicator from Merck KGaA (silica gel $60 \mathrm{~F} 254$, layer thickness $0.2 \mathrm{~mm}$ ). $\mathrm{R}_{\mathrm{f}}$-values (run level relative to the solvent front). The separations were with column chromatography at atmospheric pressure on silica gel 60 (Grain size from 0.063 to $0.200 \mathrm{~mm}$ ) from Merck KGaA. NMR spectra were recorded on a "Gemini 2000" (400/100 MHz). The ATR spectra were recorded on a FT-IR spectrometer "IFS 28" by "Bruker. The ESI mass spectra were recorded on a "Finnigan LCQ Classic". The EI mass spectra were recorded on an "Intel 402".

4.2. General procedure for the preparation of compounds $1 a-p$ : In a flask containing $5 \mathrm{ml}$ of glacial acetic acid and $2 \mathrm{mmol}$ of indole $(0.234 \mathrm{gm})$ or 5-chloroindole $0.303 \mathrm{gm}$ or 6 -chloroindole $0.303 \mathrm{gm}$ was added under stirring until all the indole was dissolved. Then $1 \mathrm{mmol}$ of the appropriate aromatic or heterocyclic aldehyde was added under vigorous stirring. The reaction mixture was allowed to stir over 4 to $6 \mathrm{~h}$, where the reaction solution turned from light yellow to light pink to dark red colour. The product was detected by TLC $\left(100 \% \mathrm{CH}_{2} \mathrm{Cl}_{2}\right)$, and when the reaction was finished $10 \mathrm{ml}$ of water were added and the solution was extracted with ethyl acetate, washed with water and $100 \mathrm{ml}$ brine, dried over anhydrous sodium sulphate and concentrated in vacuum. The product was purified by passing over a column and eluted with dichloromethane.

\subsection{Physical and Spectral Data}

3,3'-(Phenylmethylene)bis(1H-indole) (1a): $\mathrm{C}_{23} \mathrm{H}_{18} \mathrm{~N}_{2}, 322.40 \mathrm{~g} / \mathrm{mol}, \mathrm{mp} 126-127{ }^{\circ} \mathrm{C}$, pink powder, ESI-MS: $(\mathrm{m} / \mathrm{z})=321.32\left[\mathrm{M}^{+}-\mathrm{H}\right]$, IR $\left(\right.$ ATR, $\left.\mathrm{cm}^{-1}\right)=3141(\mathrm{NH}),{ }^{1} \mathrm{H}-\mathrm{NMR}:\left(400 \mathrm{MHz}\right.$, acetone- $\left.d_{6}\right) \delta(\mathrm{ppm})=5.90(\mathrm{~s}, 1 \mathrm{H}$, $\mathrm{CH}), 6.79$ (d, 2H, $J=1.5 \mathrm{~Hz}), 6.87$ (t, 2H, J=7.2 Hz), 7.04 (t, 2H, J=7.6 Hz), 7.16 (d, 1H, J=7.3 Hz), 7.25 (t, 
$2 \mathrm{H}, J=7.5 \mathrm{~Hz}), 7.32-7.39(\mathrm{~m}, 6 \mathrm{H}), 9.99(\mathrm{~s}, 2 \mathrm{H}, 2 \mathrm{NH}),{ }^{13} \mathrm{C}-\mathrm{NMR}:\left(100 \mathrm{MHz}, \mathrm{CDCl}_{3}\right) \delta(\mathrm{ppm})=40.26(\mathrm{CH})$, 110.94, 119.68, 120.59, 121.79, 121.85, 123.49, 123.99, 125.99, 126.98, 128.08, 128.59, 136.55, 143.88, EA: calcd. C, 85.68, H, 5.63, N, 8.69, found C, 85.72, H, 5.58, N, 8.66, $\mathrm{R}_{f}: 0.76\left(\mathrm{CH}_{2} \mathrm{Cl}_{2}\right)$, yield: $(580 \mathrm{mg}), 90 \%$.

3,3'(4-Nitrophenyl)methylene)bis(1 H-indole (1b): $\mathrm{C}_{23} \mathrm{H}_{17} \mathrm{~N}_{3} \mathrm{O}_{2}, 367.40 \mathrm{~g} / \mathrm{mol}, \mathrm{mp} 219-221{ }^{0} \mathrm{C}$, yellow powder, ESI-MS: $(\mathrm{m} / \mathrm{z})=366.29\left[\mathrm{M}^{+}-\mathrm{H}\right], \mathrm{IR}\left(\mathrm{ATR}, \mathrm{cm}^{-1}\right)=1456,1507\left(\mathrm{C}-\mathrm{NO}_{2}\right), 3052(\mathrm{CH}), 3455(\mathrm{NH}),{ }^{1} \mathrm{H}-$ NMR: $\left(400 \mathrm{MHz}, \mathrm{DMSO}-d_{6}\right) \delta(\mathrm{ppm})=5.98(\mathrm{~s}, 1 \mathrm{H}, \mathrm{CH}), 6.83-6.86(\mathrm{~m}, 4 \mathrm{H}), 7.02(\mathrm{~d}, 2 \mathrm{H}, J=8 \mathrm{~Hz}), 7.26(\mathrm{~d}$, $2 \mathrm{H}, J=7.9 \mathrm{~Hz}$ ), 7.35 (d, 2H, $J=8.1 \mathrm{~Hz}), 7.56$ (d, 2H, $J=8.72 \mathrm{~Hz}), 8.09$ (d, 2H, $J=8.92 \mathrm{~Hz}), 10.88$ (s, br, 2H, $2 \mathrm{NH}),{ }^{13} \mathrm{C}-\mathrm{NMR}:\left(100 \mathrm{MHz}, \mathrm{DMSO}-d_{6}\right) \delta(\mathrm{ppm})=54.82(\mathrm{CH}), 111.51,116.62,118.36,118.79,121.04$, 123.27, 123.74, 126.26, 129.32, 136.48, 145.65, 152.94, EA calcd. C, 75.19, H, 4.66, N, 11.44, found C, 75.28, $\mathrm{H}, 4.51, \mathrm{~N}, 11.60, \mathrm{R}_{f}: 0.29\left(\mathrm{CH}_{2} \mathrm{Cl}_{2}\right)$, yield: $(720 \mathrm{mg}), 98 \%$.

3,3'-((4-bromophenyl)methylene)bis(1H-indole) (1c): $\mathrm{C}_{23} \mathrm{H}_{17} \mathrm{BrN}_{2}, 401.30 \mathrm{~g} / \mathrm{mol}$, mp. $100-103{ }^{0} \mathrm{C}$, yellow crystals, ESI-MS: $(\mathrm{m} / \mathrm{z})=402\left[\mathrm{M}^{+}+\mathrm{H}\right]$, IR $\left(\right.$ ATR, $\left.\mathrm{cm}^{-1}\right)=4356(\mathrm{NH}),{ }^{1} \mathrm{H}-\mathrm{NMR}:\left(400 \mathrm{MHz}\right.$, acetone- $\left.d_{6}\right) \delta$ $(\mathrm{ppm})=5.91(\mathrm{~s}, 1 \mathrm{H}, \mathrm{CH}), 6.79(\mathrm{~d}, 2 \mathrm{H}, J=7.2 \mathrm{~Hz}), 6.87(\mathrm{t}, 2 \mathrm{H}, J=7.5 \mathrm{~Hz}), 7.07$ (t, 2H, J=7.4 Hz), $7.28(\mathrm{~d}, 2 \mathrm{H}$, $J=8 \mathrm{~Hz}), 7.36-7.40(\mathrm{~m}, 6 \mathrm{H}), 10.93(\mathrm{~s}, 2 \mathrm{H}, 2 \mathrm{NH}),{ }^{13} \mathrm{C}-\mathrm{NMR}:\left(400 \mathrm{MHz}\right.$, acetone- $\left.d_{6}\right)=57.50(\mathrm{CH}), 111.40$, $117.48,118.14,118.99,119.89,120.80,120.99,123.48,124.99,127.89,129.99,136.50,144.02, \mathrm{R}_{f} 0.65$ $\left(\mathrm{CH}_{2} \mathrm{Cl}_{2}\right)$, yield: (700 mg), $99 \%$.

3,3'-((4-Chlorophenyl)methylene)bis(1H-indole) (1d): $\mathrm{C}_{23} \mathrm{H}_{17} \mathrm{~N}_{2} \mathrm{Cl}, 356.85 \mathrm{~g} / \mathrm{mol}, \mathrm{mp} 104-106{ }^{0} \mathrm{C}$, pink powder, ESI-MS: $(\mathrm{m} / \mathrm{z})=355.11\left[\mathrm{M}^{+}-\mathrm{H}\right]$, IR $\left(\mathrm{ATR}, \mathrm{cm}^{-1}\right)=3410(\mathrm{NH}),{ }^{1} \mathrm{H}-\mathrm{NMR}:\left(400 \mathrm{MHz}, \mathrm{DMSO}-d_{6}\right) \delta$ $(\mathrm{ppm})=5.85(\mathrm{~s}, 1 \mathrm{H}, \mathrm{CH}), 6.83(\mathrm{~d}, 2 \mathrm{H}, J=7.2 \mathrm{~Hz}), 6.86(\mathrm{t}, 2 \mathrm{H}, J=7.4 \mathrm{~Hz}), 7.04(\mathrm{t}, 2 \mathrm{H}, J=7.6 \mathrm{~Hz}), 7.28(\mathrm{~d}, 2 \mathrm{H}$, $J=7.9 \mathrm{~Hz}), 7.29-7.36(\mathrm{~m}, 6 \mathrm{H}), 10.83(\mathrm{~s}, 2 \mathrm{H}, 2 \mathrm{NH}),{ }^{13} \mathrm{C}-\mathrm{NMR}:\left(100 \mathrm{MHz}, \mathrm{DMSO}-d_{6}\right)=59.65(\mathrm{CH}), 111.38$, $117.48,118.14,118.89,119.85,123.48,124.99,127.84,129.97,130.16,136.49,143.87, \mathrm{R}_{f} 0.87\left(\mathrm{CH}_{2} \mathrm{Cl}_{2}\right)$, yield: (649 mg), $76 \%$.

4-Di(1H-indol-3-yl)methyl)- $\mathbf{N}, \mathbf{N}$-dimethylaniline (1e): $\mathrm{C}_{25} \mathrm{H}_{23} \mathrm{~N}_{3}, 365.47 \mathrm{~g} / \mathrm{mol}, \mathrm{mp} .225-226{ }^{0} \mathrm{C}$, pink powder, ESI-MS: $(\mathrm{m} / \mathrm{z})=366.25\left[\mathrm{M}^{+}+\mathrm{H}\right], 364.38\left[\mathrm{M}^{+}-\mathrm{H}\right], \mathrm{IR}\left(\mathrm{ATR}, \mathrm{cm}^{-1}\right)=3314(\mathrm{NH}),{ }^{1} \mathrm{H}-\mathrm{NMR}:(400 \mathrm{MHz}$, DMSO- $\left.d_{6}\right) \delta(\mathrm{ppm})=4.60\left(\mathrm{~s}, \mathrm{br} ., 6 \mathrm{H}, 2 \mathrm{CH}_{3}\right), 5.89(\mathrm{~s}, 1 \mathrm{H}, \mathrm{CH}), 6.84-6.88(\mathrm{~m}, 4 \mathrm{H}), 7.03(\mathrm{t}, 2 \mathrm{H}, J=7.99 \mathrm{~Hz})$, 7.28 (d, 2H, $J=7.9 \mathrm{~Hz}), 7.34$ (d, 2H, $J=8.1 \mathrm{~Hz}), 7.49$ (t, 4H, $J=10.6 \mathrm{~Hz}), 10.84$ (s, 2H, 2NH), ${ }^{13} \mathrm{C}-\mathrm{NMR}:(100$ $\left.\mathrm{MHz}, \mathrm{DMSO}-d_{6}\right) \delta(\mathrm{ppm})=40.13\left(\mathrm{CH}_{3}\right), 43.62\left(\mathrm{CH}_{3}\right), 45.07(\mathrm{CH}), 111.39,114.52,117.43,118.13,118.85$, 119.08, 120.83, 121.40, 123.47, 124.23, 126.37, 129.47, 136.46, 141.84, EA: calcd. C, 82.16; H, 6.34; N, 11.50, found $\mathrm{C}, 82.20, \mathrm{H}, 6.37, \mathrm{~N}, 11.53, \mathrm{R}_{f} 0.29\left(\mathrm{CH}_{2} \mathrm{Cl}_{2}\right)$, yield: $(665 \mathrm{mg}), 91 \%$.

3,3'-((3-Bromophenyl)methylene)bis(1H-indole) (1f): $\mathrm{C}_{23} \mathrm{H}_{17} \mathrm{BrN}_{2}, 401.30 \mathrm{~g} / \mathrm{mol}, \mathrm{mp} .93$ - $95{ }^{0} \mathrm{C}$, red crystals, ESI-MS: $(\mathrm{m} / \mathrm{z})=401.26\left[\mathrm{M}^{+}+\mathrm{H}\right], 399.31\left[\mathrm{M}^{+}-\mathrm{H}\right]$, IR: $\left(\mathrm{ATR}, \mathrm{cm}^{-1}\right)=3405(\mathrm{NH}),{ }^{1} \mathrm{H}-\mathrm{NMR}:(400$ $\left.\mathrm{MHz}, \mathrm{DMSO}-d_{6}\right) \delta(\mathrm{ppm})=5.86(\mathrm{~s}, 1 \mathrm{H}, \mathrm{CH}), 6.85-6.86(\mathrm{~m}, 3 \mathrm{H}), 7.03(\mathrm{t}, 2 \mathrm{H}, J=7.6 \mathrm{~Hz}), 7.22(\mathrm{t}, 1 \mathrm{H}, J=7.8$ $\mathrm{Hz}), 7.28$ (d, 2H, J=7.9 Hz), $7.34-7.37$ (m, 5H), 7.49 (s, 1H), 10.84 (s, 2H, 2NH), ${ }^{13} \mathrm{C}-\mathrm{NMR}$ : (100 MHz, DMSO- $\left.d_{6}\right) \delta(\mathrm{ppm})=39.16(\mathrm{CH}), 111.38,117.20,118.16,118.80,120.84,121.25,123.51,126.32,127.23$, 128.54, 130.08, 130.69, 136.42, 147.78, EA: calcd. C, 68.84, H, 4.27, Br, 19.91, N, 6.98, found C, 68.90, H, 4.30, $\mathrm{Br}, 19.95, \mathrm{~N}, 7.00, \mathrm{R}_{f}$-Value: $0.74\left(\mathrm{CH}_{2} \mathrm{Cl}_{2}\right)$, yield: $(787 \mathrm{mg}), 88 \%$.

3,3'(3-Benzyloxy)phenyl)methylene)bis(1H-indole (1g): $\mathrm{C}_{30} \mathrm{H}_{24} \mathrm{~N}_{2} \mathrm{O}, 428.52 \mathrm{~g} / \mathrm{mol}$, mp. 190 - $192{ }^{0} \mathrm{C}$, white powder, ESI-MS: $(\mathrm{m} / \mathrm{z})=428.24\left[\mathrm{M}^{+}-\mathrm{H}\right]$, IR $\left(\right.$ ATR, $\left.\mathrm{cm}^{-1}\right)=1262(\mathrm{C}-\mathrm{O}), 2852,3034(\mathrm{CH}), 3425(\mathrm{NH}),{ }^{1} \mathrm{H}-$ NMR: $\left(400 \mathrm{MHz}\right.$, acetone- $\left.d_{6}\right) \delta(\mathrm{ppm})=5.01\left(\mathrm{~s}, 2 \mathrm{H}, \mathrm{CH}_{2}\right), 5.90(\mathrm{~s}, 1 \mathrm{H}, \mathrm{CH}), 6.82(\mathrm{~d}, 2 \mathrm{H}, J=7.5 \mathrm{~Hz}), 6.85(\mathrm{~d}$, 2H, J=7.2 Hz), 6.90 (t, 2H, J=7.5 Hz), 7.00 - 7.11 (m, 4H), 7.18 (t, 1H, J=7.9 Hz), 7.26 - 7.33 (m, 2H), 7.37 $7.39(\mathrm{~m}, 6 \mathrm{H}), 9.95$ (s, br., $2 \mathrm{H}, 2 \mathrm{NH}),{ }^{13} \mathrm{C}-\mathrm{NMR}$ : $\left(100 \mathrm{MHz}\right.$, acetone- $\left.d_{6}\right) \delta(\mathrm{ppm})=41.18(\mathrm{CH}), 70.31\left(\mathrm{CH}_{2}-\mathrm{O}\right)$, $112.06,112.91,116.41,119.26,119.63,120.21,121.98,122.13,123.51,124.45,128.04,128.32$, 128.37, 
129.07, 129.23, 129.69, 137.98, 138.38, 147.55, 159.66 , EA: calcd. C, 84.08; H, 5.65; N, 6.54, found C, 84.12, H, 5.55, N, 6.58, $\mathrm{R}_{f}: 0.79\left(\mathrm{CH}_{2} \mathrm{Cl}_{2}\right)$, yield: (746 mg), $87 \%$.

4-(Di(1H-indol-3-yl)methyl)benzene-1,2-diol (1h): $\mathrm{C}_{23} \mathrm{H}_{18} \mathrm{~N}_{2} \mathrm{O}_{2}, 354.40 \mathrm{~g} / \mathrm{mol}$, mp. $105-107{ }^{0} \mathrm{C}$, light brown powder, ESI-MS: $(\mathrm{m} / \mathrm{z})=392.89\left[\mathrm{M}^{+}+\mathrm{K}\right], 354.25\left[\mathrm{M}^{+}\right], 353.24\left[\mathrm{M}^{+}-\mathrm{H}\right]$, IR: $\left(\mathrm{ATR}, \mathrm{cm}^{-1}\right)=1215(\mathrm{C}-\mathrm{O}), 2922$, $3051(\mathrm{CH}), 3400(\mathrm{NH}),{ }^{1} \mathrm{H}-\mathrm{NMR}$ : $\left(400 \mathrm{MHz}\right.$, acetone- $\left.d_{6}\right) \delta(\mathrm{ppm})=5.77(\mathrm{~s}, 1 \mathrm{H}, \mathrm{CH}), 6.45(\mathrm{~s}, 1 \mathrm{H}), 6.76(\mathrm{~d}$, 2H, J=8.9 Hz), $6.86-6.89$ (m, 2H), 7.04 (s, 2H), 7.29 (s, 1H), 7.35 (s, 4H), 7.55 (s, 1H), 9.89 (s, 2H, 2NH), EA: calcd. C, 77.95; H, 5.12; N, 7.90, found C, 78.01, H, 5.20, N, 7.96, $\mathrm{R}_{f .} 0.62\left(\mathrm{CH}_{2} \mathrm{Cl}_{2}\right)$, yield: $(517 \mathrm{mg}), 73$ $\%$.

3,3-(3-Benzyloxy)-4-methoxyphenyl)methylene)bis(1H-indole (1i): $\mathrm{C}_{31} \mathrm{H}_{26} \mathrm{~N}_{2} \mathrm{O}_{2}, 458.55 \mathrm{~g} / \mathrm{mol}$, mp 75 - 78 ${ }^{0} \mathrm{C}$, orange crystals, ESI-MS: $(\mathrm{m} / \mathrm{z})=481.16\left[\mathrm{M}^{+}+\mathrm{Na}\right], 457.24\left[\mathrm{M}^{+}-\mathrm{H}\right]$, IR $\left(\right.$ ATR, $\left.\mathrm{cm}^{-1}\right)=1262(\mathrm{C}-\mathrm{O}), 2850$, $2925(\mathrm{CH}), 3398(\mathrm{NH}),{ }^{1} \mathrm{H}-\mathrm{NMR}:\left(400 \mathrm{MHz}, \mathrm{DMSO}-d_{6}\right) \delta(\mathrm{ppm})=3.71(\mathrm{~s}, 3 \mathrm{H}, \mathrm{OMe}), 4.95\left(\mathrm{~s}, 2 \mathrm{H}, \mathrm{CH}_{2}\right), 5.71$ (s, 1H, CH), $6.74-6.76(\mathrm{~m}, 2 \mathrm{H}), 6.81-6.86(\mathrm{~m}, 4 \mathrm{H}), 7.02(\mathrm{t}, 2 \mathrm{H}, J=7.5 \mathrm{~Hz}), 7.06(\mathrm{~s}, 1 \mathrm{H}), 7.23(\mathrm{~d}, 2 \mathrm{H}, J=7.9$ $\mathrm{Hz}), 7.29-7.31(\mathrm{~m}, 6 \mathrm{H}), 7.34(\mathrm{~s}, 1 \mathrm{H}), 10.73(\mathrm{~s}, 2 \mathrm{H}, 2 \mathrm{NH}),{ }^{13} \mathrm{C}-\mathrm{NMR}:\left(100 \mathrm{MHz}, \mathrm{DMSO}-d_{6}\right) \delta(\mathrm{ppm})=55.59$ (CH), $59.70(\mathrm{OMe}), 70.08\left(\mathrm{OCH}_{2}\right), 111.29,111.98,114.94,118.00,118.24,119.03,120.71,123.29,126.24$, 126.56, 127.63, 127.75, 127.86, 128.18, 128.35, 136.49, 137.09, 137.39, 147.14, 147.38, EA: calcd. C, 81.20; $\mathrm{H}, 5.72 ; \mathrm{N}, 6.11$, found $\mathrm{C}, 81.22, \mathrm{H}, 5.75, \mathrm{~N}, 6.14, \mathrm{R}_{f} 0.79\left(\mathrm{CH}_{2} \mathrm{Cl}_{2}\right)$, yield: $(816 \mathrm{mg}), 89 \%$.

3,3-((4-Benzyloxy)-3-methoxyphenyl)methylene)bis(1H-indole (1j): $\mathrm{C}_{31} \mathrm{H}_{26} \mathrm{~N}_{2} \mathrm{O}_{2}, 458.55 \mathrm{~g} / \mathrm{mol}, \mathrm{mp} 215$ $219{ }^{0} \mathrm{C}$, light orange crystals, ESI-MS: $(\mathrm{m} / \mathrm{z})=457.20\left[\mathrm{M}^{+}-\mathrm{H}\right]$, IR: $\left(\right.$ ATR, $\left.\mathrm{cm}^{-1}\right)=1245(\mathrm{C}-\mathrm{O}), 2961,3036$ $(\mathrm{CH}), 3416(\mathrm{NH}),{ }^{1} \mathrm{H}-\mathrm{NMR}:\left(400 \mathrm{MHz}\right.$, acetone- $\left.d_{6}\right) \delta(\mathrm{ppm})=3.70(\mathrm{~s}, 3 \mathrm{H}, \mathrm{OMe}), 5.04\left(\mathrm{~s}, 2 \mathrm{H}, \mathrm{CH}_{2}\right), 5.85(\mathrm{~s}$, 1H, CH), $6.81(\mathrm{~s}, 2 \mathrm{H}), 6.85-6.92(\mathrm{~m}, 4 \mathrm{H}), 7.04$ (t, 2H, J=7.6 Hz), 7.09 (s, 1H), 7.29 (d, 1H, J=7.5 Hz), 7.33 7.37 (m, 6H), 7.47 (d, 2H, J=7.7 Hz), $9.95(\mathrm{~s}, 2 \mathrm{H}, 2 \mathrm{NH}),{ }^{13} \mathrm{C}-\mathrm{NMR}$ : $\left(400 \mathrm{MHz}\right.$, acetone- $\left.d_{6}\right) \delta(\mathrm{ppm})=40.75$ $(\mathrm{CH}), 56.19$ (OMe), $71.61\left(\mathrm{OCH}_{2}\right), 112.05,112.10,114.33,114.93,119.23,120.09,120.32,121.47,121.98$, 124.32, 124.47, 128.12, 128.45, 128.49, 129.12, 138.08, 138.83, 139.31, 147.72, 150.64, EA: calcd. C, 81.20; $\mathrm{H}, 5.72 ; \mathrm{N}, 6.11$, found $\mathrm{C}, 81.02, \mathrm{H}, 5.90, \mathrm{~N}, 6.22, \mathrm{R}_{f}: 0.71\left(\mathrm{CH}_{2} \mathrm{Cl}_{2}\right)$, yield: $(844 \mathrm{mg}), 92 \%$.

2,4,6-(3,3'-(Trifluoro-3-methylphenyl)methylene)bis(1H-indole) (1k): $\mathrm{C}_{24} \mathrm{H}_{17} \mathrm{~F}_{3} \mathrm{~N}_{2}, 390.40 \mathrm{~g} / \mathrm{mol}$, mp. $>350$ ${ }^{0} \mathrm{C}$, white powder, ESI-MS: $(\mathrm{m} / \mathrm{z})=391.90\left[\mathrm{M}^{+}+\mathrm{H}\right], 389.31\left[\mathrm{M}^{+}-\mathrm{H}\right]$, IR-Spectrum: $\left(A T R, \mathrm{~cm}^{-1}\right)=2960,3055$ $(\mathrm{CH}), 3443(\mathrm{NH}),{ }^{1} \mathrm{H}-\mathrm{NMR}:\left(400 \mathrm{MHz}, \mathrm{DMSO}-d_{6}\right) \delta(\mathrm{ppm})=3.15(\mathrm{~s}, 3 \mathrm{H}, \mathrm{Me}), 5.73(\mathrm{~s}, 1 \mathrm{H}, \mathrm{CH}), 6.86(\mathrm{t}, 1 \mathrm{H}$, $J=10.9 \mathrm{~Hz}), 6.99-7.14(\mathrm{~m}, 2 \mathrm{H}), 7.19(\mathrm{~d}, 1 \mathrm{H}, J=8.2 \mathrm{~Hz}), 7.21-7.29(\mathrm{~m}, 2 \mathrm{H}), 7.35$ (t, 1H, J=7.7 Hz), 7.44 (s, $1 \mathrm{H}), 7.66(\mathrm{~d}, 1 \mathrm{H}, J=8.2 \mathrm{~Hz}), 7.74$ (t, 2H, $J=10.4 \mathrm{~Hz}), 8.37$ (s, 2H, 2NH), ${ }^{13} \mathrm{C}-\mathrm{NMR}$ : $\left(100 \mathrm{MHz}, \mathrm{DMSO}-d_{6}\right) \delta$ $(\mathrm{ppm})=38.87(\mathrm{Me}), 52.77(\mathrm{CH}), 109.00,110.02,117.32,119.88,120.17,122.73,126.12,126.21,127.37$, 128.25, 128.78, 129.21, 134.22, 142.00, EA: calcd. C, 73.84; H, 4.39; F, 14.60; N, 7.18, found C, 74.01, H, 4.52, F, 14.52, N, 7.23, $\mathrm{R}_{f}: 0.71\left(\mathrm{CH}_{2} \mathrm{Cl}_{2}\right)$, yield: $(390 \mathrm{mg}), 77 \%$.

3,3-(Naphthalen-1-ylmethylene)bis(1H-indole (1l): $\mathrm{C}_{27} \mathrm{H}_{20} \mathrm{~N}_{2}, 372.46 \mathrm{~g} / \mathrm{mol}, \mathrm{mp}: 252-255{ }^{0} \mathrm{C}$, White powder, ESI-MS: $(\mathrm{m} / \mathrm{z})=371.30\left[\mathrm{M}^{+}-\mathrm{H}\right]$, IR: $\left(\mathrm{ATR}, \mathrm{cm}^{-1}\right)=2834,3048(\mathrm{CH}), 3407(\mathrm{NH}),{ }^{1} \mathrm{H}-\mathrm{NMR}:(400$ MHz, DMSO- $\left.d_{6}\right) \delta(\mathrm{ppm})=5.71(\mathrm{~s}, 1 \mathrm{H}, \mathrm{CH}), 6.59(\mathrm{~s}, 1 \mathrm{H}), 6.68(\mathrm{~d}, 2 \mathrm{H}, J=7 \mathrm{~Hz}), 6.81(\mathrm{t}, 2 \mathrm{H}, J=7.5 \mathrm{~Hz}), 6.99$ (t, 2H, $J=7.6 \mathrm{~Hz}), 7.23(\mathrm{~d}, 4 \mathrm{H}, J=8.1 \mathrm{~Hz}), 7.32(\mathrm{t}, 2 \mathrm{H}, J=9 \mathrm{~Hz}), 7.41(\mathrm{t}, 2 \mathrm{H}, J=7.7 \mathrm{~Hz}), 7.73(\mathrm{~d}, 1 \mathrm{H}, J=8 \mathrm{~Hz})$, $7.88(\mathrm{~d}, 1 \mathrm{H}, J=7.5 \mathrm{~Hz}), 8.22(\mathrm{~d}, 1 \mathrm{H}, J=8 \mathrm{~Hz}), 10.74(\mathrm{~s}, 2 \mathrm{H}, 2 \mathrm{NH}),{ }^{13} \mathrm{C}-\mathrm{NMR}:\left(100 \mathrm{MHz}\right.$, DMSO-$\left.d_{6}\right) \delta(\mathrm{ppm})=$ $35.33(\mathrm{CH}), 111.41,117.62,118.15,118.84,120.77,123.84,124.13,125.15,125.19,125.42,125.68,126.43$, 126.54, 128.42, 131.23, 133.49, 136.56, 140.18, EA. calcd. C, 87.07; H, 5.41; N, 7.52, found C, 87.00, H, 5.51, N, 7.55, $\mathrm{R}_{f}: 0.87\left(\mathrm{CH}_{2} \mathrm{Cl}_{2}\right)$, yield: (722 mg), $97 \%$.

3,3-(Pyridin-3-ylmethylene)bis(1H-indole $(\mathbf{1 m}): \mathrm{C}_{22} \mathrm{H}_{17} \mathrm{~N}_{3}, 323.39 \mathrm{~g} / \mathrm{mol}, \mathrm{mp} 98-101{ }^{0} \mathrm{C}$, light pink powder, ESI-MS: $(\mathrm{m} / \mathrm{z})=324.16\left[\mathrm{M}^{+}+\mathrm{H}\right]$, IR $\left(\mathrm{ATR}, \mathrm{cm}^{-1}\right)=2917,3055(\mathrm{CH}), 3403(\mathrm{NH}),{ }^{1} \mathrm{H}-\mathrm{NMR}:(400 \mathrm{MHz}$, DMSO- $\left.d_{6}\right) \delta(\mathrm{ppm})=5.70(\mathrm{~s}, 1 \mathrm{H}, \mathrm{CH}), 5.88(\mathrm{~s}, 1 \mathrm{H}, \mathrm{CH}), 6.84(\mathrm{t}, 4 \mathrm{H}, J=7.1 \mathrm{~Hz}), 7.01(\mathrm{t}, 2 \mathrm{H}, J=7.6 \mathrm{~Hz}), 7.22$ - 
$7.29(\mathrm{~m}, 3 \mathrm{H}), 7.32(\mathrm{~d}, 2 \mathrm{H}, J=8.1 \mathrm{~Hz}), 7.65(\mathrm{~d}, 1 \mathrm{H}, J=7.9 \mathrm{~Hz}), 8.34-8.37(\mathrm{~m}, 1 \mathrm{H}), 8.58(\mathrm{~d}, 1 \mathrm{H}, J=7.9 \mathrm{~Hz})$, $10.84(\mathrm{~s}, 2 \mathrm{H}, 2 \mathrm{NH}),{ }^{13} \mathrm{C}-\mathrm{NMR}:\left(100 \mathrm{MHz}, \mathrm{DMSO}-d_{6}\right) \delta(\mathrm{ppm})=54.79(\mathrm{CH}), 54.78(\mathrm{CH}), 111.45,117.07$, 118.24, 118.83, 120.94, 123.15, 123.56, 126.29, 135.47, 136.51, 140.15, 146.99, 149.50, EA: calcd. C, 81.71; $\mathrm{H}, 5.30 ; \mathrm{N}, 2.99$, found $\mathrm{C}, 81.90, \mathrm{H}, 5.35, \mathrm{~N}, 13.02, \mathrm{R}_{f} 0.46\left(7 \% \mathrm{MeOH} / \mathrm{CH}_{2} \mathrm{Cl}_{2}\right)$, yield: $(614 \mathrm{mg}), 95 \%$.

Tri(1H-indol-3-yl)methane (1n): $\mathrm{C}_{25} \mathrm{H}_{19} \mathrm{~N}_{3}, 361.44 \mathrm{~g} / \mathrm{mol}, \mathrm{mp}: 235-240{ }^{0} \mathrm{C}$, light yellow powder, ESI-MS: $(\mathrm{m} / \mathrm{z})=360.32\left[\mathrm{M}^{+}-\mathrm{H}\right]$, IR $\left(\mathrm{ATR}, \mathrm{cm}^{-1}\right)=2882,3054(\mathrm{CH}), 3424(\mathrm{NH}),{ }^{1} \mathrm{H}-\mathrm{NMR}$ : $\left(400 \mathrm{MHz}\right.$, acetone- $\left.d_{6}\right) \delta$ $(\mathrm{ppm})=6.19(\mathrm{~s}, 1 \mathrm{H}, \mathrm{CH}), 6.85-6.93(\mathrm{~m}, 6 \mathrm{H}), 7.03(\mathrm{t}, 4 \mathrm{H}, J=7.6 \mathrm{~Hz}), 7.37(\mathrm{t}, 3 \mathrm{H}, J=7.8 \mathrm{~Hz}), 7.48(\mathrm{t}, 2 \mathrm{H}$, $J=7.4 \mathrm{~Hz}), 9.88(\mathrm{~s}, 3 \mathrm{H}, 3 \mathrm{NH}),{ }^{13} \mathrm{C}-\mathrm{NMR}:\left(100 \mathrm{MHz}\right.$, acetone- $\left.d_{6}\right) \delta(\mathrm{ppm})=31.33(\mathrm{CH}), 111.13,118.95$, 119.08, 120.12, 121.09, 123.17, 124.60, 127.35, 128.17, 137.19, Elemental analysis: Calcd. C, 83.08; H, 5.30; N, 11.63, found $\mathrm{C}, 83.09, \mathrm{H}, 5.33, \mathrm{~N}, 11.71, \mathrm{R}_{f}-$ Value: $0.73\left(\mathrm{CH}_{2} \mathrm{Cl}_{2}\right)$, yield: $(708 \mathrm{mg}), 98 \%$.

3,3-((3-Benzyloxy)-4-methoxyphenyl)methylene)bis(5-chloro-1H-indole (1o): $\mathrm{C}_{31} \mathrm{H}_{24} \mathrm{Cl}_{2} \mathrm{~N}_{2} \mathrm{O}_{2}, 527.44 \mathrm{~g} / \mathrm{mol}$, mp. $82-85^{\circ} \mathrm{C}$, ESI-MS: $(\mathrm{m} / \mathrm{z})=528.18\left[\mathrm{M}^{+}+\mathrm{H}\right]$, IR $\left(\mathrm{ATR}, \mathrm{cm}^{-1}\right)=1259(\mathrm{C}-\mathrm{O}), 2850,2924(\mathrm{CH}), 3369(\mathrm{NH})$, ${ }^{1} \mathrm{H}-\mathrm{NMR}:\left(400 \mathrm{MHz}, \mathrm{CDCl}_{3}\right) \delta(\mathrm{ppm})=3.77(\mathrm{~s}, 3 \mathrm{H}, \mathrm{OMe}), 4.93\left(\mathrm{~s}, 2 \mathrm{H}, \mathrm{CH}_{2}\right), 5.52(\mathrm{~s}, 2 \mathrm{H}, \mathrm{CH}), 6.41(\mathrm{~d}, 2 \mathrm{H}$, $J=7.6 \mathrm{~Hz}), 6.73(\mathrm{t}, 4 \mathrm{H}, J=7.3 \mathrm{~Hz}), 7.02(\mathrm{~d}, 2 \mathrm{H}, J=7 \mathrm{~Hz}), 7.13(\mathrm{~d}, 2 \mathrm{H}, J=8.6 \mathrm{~Hz}), 7.18$ (dd, 6H, $J=3.1,7.1 \mathrm{~Hz})$, $7.88(\mathrm{~s}, 2 \mathrm{H}, 2 \mathrm{NH}),{ }^{13} \mathrm{C}-\mathrm{NMR}:\left(100 \mathrm{MHz}\right.$, acetone- $\left.d_{6}\right) \delta(\mathrm{ppm})=39.39(\mathrm{CH}), 55.99(\mathrm{OMe}), 71.03\left(\mathrm{OCH}_{2}\right)$, $111.51,111.76,112.12,115.37,119.15,121.20,122.31,124.77,124.99,126.91,127.46,127.50,127.66$, 127.96, 128.64, 135.04, 135.74, 137.10, 147.63, 148.36, EA: calcd. C, 70.59; H, 4.59; Cl, 13.44; N, 5.31, found $\mathrm{C}, 70.62, \mathrm{H}, 4.55, \mathrm{Cl}, 13.55, \mathrm{~N}, 5.51, \mathrm{R}_{f}: 0.68\left(\mathrm{CH}_{2} \mathrm{Cl}_{2}\right)$, yield: $(960 \mathrm{mg}), 91 \%$.

3,3/-((3-(Benzyloxy)-4-methoxyphenyl)methylene)bis(6-chloro-1H-indole (1p): $\quad \mathrm{C}_{31} \mathrm{H}_{24} \mathrm{Cl}_{2} \mathrm{~N}_{2} \mathrm{O}_{2}, \quad 527.44$ $\mathrm{g} / \mathrm{mol}$, mp. $85-87{ }^{\circ} \mathrm{C}$, light orange crystals, ESI-MS: $(\mathrm{m} / \mathrm{z})=526.14\left[\mathrm{M}^{+}-\mathrm{H}\right]$, IR $\left(\mathrm{ATR}, \mathrm{cm}^{-1}\right)=1253(\mathrm{C}-\mathrm{O})$, 2866, $2928(\mathrm{CH}), 3420(\mathrm{NH}),{ }^{1} \mathrm{H}-\mathrm{NMR}:\left(400 \mathrm{MHz}, \mathrm{DMSO}-d_{6}\right) \delta(\mathrm{ppm})=3.70(\mathrm{~s}, 3 \mathrm{H}, \mathrm{OMe}), 4.94(\mathrm{~s}, 2 \mathrm{H}$, $\left.\mathrm{OCH}_{2}\right), 5.69(\mathrm{~s}, 1 \mathrm{H}, \mathrm{CH}), 6.77$ (d, 2H, $\left.J=2 \mathrm{~Hz}\right), 6.79$ (d, 1H, $\left.J=1.9 \mathrm{~Hz}\right), 6.84$ (t, 2H, J=7.9 Hz), 7.00 (d, 1H, $J=2 \mathrm{~Hz}), 7.17$ (d, 2H, $J=8.6 \mathrm{~Hz}), 7.30$ (t, H, $J=5.7 \mathrm{~Hz}), 7.37$ (d, 2H, $J=1.6 \mathrm{~Hz}), 10.91(\mathrm{~s}, 2 \mathrm{H}, 2 \mathrm{NH}),{ }^{13} \mathrm{C}-\mathrm{NMR}$ : $\left(100 \mathrm{MHz}, \mathrm{DMSO}-d_{6}\right) \delta(\mathrm{ppm})=26.78(\mathrm{CH}), 55.99(\mathrm{OMe}), 70.41\left(\mathrm{OCH}_{2}\right), 111.47,112.41,115.11,118.79$, $118.95,120.83,121.08,125.02,125.79,126.10,127.02,128.17,128.29,128.71,128.89,137.22,137.40$, 137.59, 147.70, 147.99, Elemental analysis: calcd. C, 70.59; H, 4.59; Cl, 13.44; N, 5.31, found C, 70.63, H, 4.72, $\mathrm{Cl}, 13.53, \mathrm{~N}, 5.34, \mathrm{R}_{f} 0.68\left(\mathrm{CH}_{2} \mathrm{Cl}_{2}\right)$, yield: $(960 \mathrm{mg}), 93 \%$.

\section{References}

1. (a) Morteza S, Mohammad Z., Hendrik G., Zahra T. (2010) Bis- and trisindolylmethanes (BIMs and TIMs), Chem Rev. 110(4), 2250-2293. (b) Maciejewska, D.; Szpakowska, I.; Wolska, I.; Niemyjska, M.;Mascini, M.; Maj-Zurawska, M. (2006) DNA-based electrochemical biosensors for monitoring of bisindoles as potential antitumoral agents, chemistry, X-ray crystallography, Bio electrochemistry, 69,1-9.

2. Maciejewska, D.; Niemyjska, M.; Wolska, I.; Waostowski, M.;Rasztawicka, M. (2004) Synthesis, Spectroscopic Studies and Crystal Structure of 5,5'-Dimethoxy-3,3'-methanediyl-bis-indole as the Inhibitor of Cell Proliferation of Human Tumors, Z. Naturforsch., B: Chem. Sci.59, 1137-1142.

3. Maciejewska, D.; Wolska, I.; Niemyjska, M.; Zero, P. (2005) Structure in solid state of 3,30diindolylmethane derivatives, potent cytotoxic agents against human tumor cells, followed X-ray diffraction and 13C CP/MAS NMR analyses, J. Mol. Struct. 753, 53-60.

4. Mason, M. R.; Fneich, B. N.; Kirschbaum, K. (2003) Titanium and Zirconium Amido Complexes Ligated by 2,2'-Di(3-methylindolyl)methanes: Synthesis, Characterization, and Ethylene Polymerization Activity. Inorg. Chem. 42, 6592-6594.

5. Mason, M. R. (2003) Di- and Triindolylmethanes: Versatile Ligands for Main Group and Transition Elements, Chemtracts, 16, 272-289.

6. Barnard, T. S.; Mason, M. R. (2001) Hindered Axial-Equatorial Carbonyl Exchange in an Fe(CO)4(PR3) Complex of a Rigid Bicyclic Phosphine, Inorg. Chem. 40, 5001-5009.

7. Barnard, T. S.; Mason, M. R. (2001) Synthesis, Structure and Coordination Chemistry of the Bicyclic pAcid Phosphatri(3-methylindolyl)methane, Organometallics, 20, 206-214. 
8. Tanski, J. M.; Parkin, G. (2003) Tantalum Amido and Imido Complexes Supported by Tris[(2indolyl)methyl]amine, a Tetradentate Trianionic Ligand with Reduced $\partial$-Donor Character, Inorg. Chem. 42, 264-266.

9. Black, D. S. (1993) New Dimensions in Indole Chemistry from Ligand Design to Natural Products Synlett 246-252.

10. Raju, B. C.; Rao, J. M.,(2008) An Easy and Efficient Synthesis of Bisindolylmethanes and Tetraindolylmethane Tröger's Base Catatlyzed by $\mathrm{AgBF}_{4}$, Indian J. Chem. 47B, 899-905.

11. Sujatha, K.; Perumal, P. T.; Muralidharan, D.; Rajendran, M. (2009) Synthesis and Biological Evaluation of some novel Heterocyclic Derivatives of Paraacetamole as Potential Analgesic and Antipyretic Agents, Indian J. Chem. 48B, 267-269.

12. Nagarajan, R.; Perumal, P. T. (2004) Expedient Synthesis of Bis-indolylmethanes, Chem. Lett. 33, 150159.

13. Chakrabarty, M.; Mukherjee, R.; Mukherji, A.; Arima, S.; Harigaya, Y. (2006) Phosphoric Acid-on-Silica Gel: A Green Catalyst for the Synthesis of Symmetrical Bis(indolyl)alkanes, Heterocycle 68, 1659-1668.

14. Yu, L.; Chen, D.; Li, J.; Wang, P. G.(1997) Preparation, Characterization, and Synthetic Uses of Lanthanide(III) Catalysts Supported on Ion Exchange Resins, J. Org. Chem. 62, 3575-3581.

15. Karthik, M.; Tripathi, A. K.; Gupta, N. M.; Palanichamy, M.; Murugesan, V. (2004) A highly efficient, eco-friendly, room temperature synthesis of bis(indol-3-yl)methanes using the mesoporous titanosilicate Ti-TUD-1: electrophilic substitution reactions of indoles Part XXIII Catal. Commun. 5, 209-216.

16. Penieres-Carrillo, G.; Garcı'a-Estrada, J. G.; Gutie'rrez-Ramı'reza, J. L.; Alvarez-Toledanob, C. (2003) Infrared-assisted eco-friendly selective synthesis of diindolylmethanes Green Chem.5, 337-339.

17. Maiti, A. K.; Bhattacharyya, P. (1997) Montmorillonite Clay-catalysed Synthesis of Bis(indol-3-yl)methanes and 1,2-Bis(indol-3-yl)ethanes J. Chem. Res. 424-425.

18. Banerji, J.; Dutta, U.; Basak, B.; Saha, M.; Budzikiewicz, H.; Chatterjee, A.(2001) A facile $\mathrm{Ph}_{3} \mathrm{P} / \mathrm{CO}_{2}$ mediated, one-pot synthesis of 2-oxazolidinones from 1,2-azido alcohols via phosphazene and isocyanate intermediates Indian J. Chem. 40B, 978-984.

19. Ramesh, C.; Ravindranath, N.; Das, B. (1999) Coordination Networks with 1,3-Bis(4-pyridyl)propane. A Flexible Ligand Exhibiting Supramolecular Isomerism J. Chem. Res. (S) 72. DOI: 10.1039/a805819i

20. Nagawade, R. R.; Shinde, D. B., (2005) PCL5 as A Mild and Efficient Catalyst for the Synthesis of Bisindolylmethanes and Di-bisindolylmethanes Bull. Korean Chem. Soc. 26, 453-458. .

21. Bandgar, B. P.; Shaikh, K. A.(2004) $\mathrm{SnCl}_{2 \cdot 2} \mathrm{H}_{2} \mathrm{O}$; a precious addition to catalyst range for synthesis of bis(indolyl)methanes $J$. Chem. Res. 34, 730-736.

22. Mohammadpoor-Baltork, I.; Memarian, H. R.; Khosropour, A. R.; Nikoofar, K. (2006) BiOClO ${ }_{4} \cdot \mathrm{xH}_{2} \mathrm{O}$ and $\mathrm{Bi}(\mathrm{OTf})_{3}$ as Efficient and Environmentally Benign Catalysts for Synthesis of Bis(indolyl)methanes in Solution and Under Ultrasound Irradiation Lett. Org. Chem.3, 768-772.

23. Thirupathi Reddy, Y.; Narsimha Reddy, P.; Sunil Kumar, B.; Rajitha, B. (2005) Efficient synthesis of bisindolylmethanes catalyzed by TiCl4 Indian, J. Chem. 44B, 2393-2395.

24. Kamal, A.; Khan, M. N. A.; Reddy, K. S.; Srikanth, Y. V. V.; Ahmed, S. K.; Kumar, K. P.; Murthy, U. S. N. (2009) An efficient synthesis of bis(indolyl)methanes and evaluation of their antimicrobial activities $J$. Enzyme Inhib. Med. Chem 24, 559-565.

25. Kruber (1941) Isolated from Isolierung aus Steinkohlenteer Chem. Ber 74, 1688 - 1692.

26. (a): Segall, A.; Pappa, H.; Pizzorno, M. T.; Radice, M.; Amoroso, A.; Gutkind, G. O. (1996) antimicrobial activity of 5,6-Dihydrobenzo-[A]-carbazoles .2. Farmaco Ed. Sci. 51, 513 - 516. (b): Pappa, H.; Segall, A.; Pizzorno, M. T.; Radice, M.; Amoroso, A.; Gutkind, G. O. (1994) Antimicrobial Activity of 5,6Dihydrobenzo-[A]-carbaoles Farmaco Ed. Sci.49, 333 - 336.

27. Amoroso, A.; Radice, M.; Segall, A.; Rodero, L.; Hochenfellner, F.; Pizzorno, M. T.; Moretton, J.; Garrido, D.;Gutkind, G. (2000) Antitumoral activity of trisubstituted dihydrobenzo(a)carbazoles Pharmazie 55, 151 - 152.

28. Segall, A.; Pizzorno, M. T. (2000) Antitumoral activity of trisubstituted dihydrobenzo(a)carbazoles. Part III Pharmazie 55, 766 - 767.

29. Golob, T.; Biberger, C.; Walter, G.; Angerer, E. (2000) Antiestrogenic activities of 3,8-dihydroxy-6,11dihydrobenzo[a]carbazoles with sulfur-containing side chains Arch.Pharm. 2000, 333, 305 - 311.

30. Frederich, M.; Jacquier, M.-J.; Thepenier, P.; Mol, P. D.; Tits, M.; Philippe, G.; Delaude, C.; Angenot, L.; Zeches-Hanrot, M. (2002) Antiamoebic and antiplasmodial activities of alkaloids ... alkaloids from Strychnos icaja. J. Nat. Prod. 65, 1381 - 1386. 
31. Fertuck, K. C.; Kumar, S.; Sikka, H. C.; Matthews, J. B.;Zacharewski, T. R., (2001) Interaction of PAHrelated compounds with the isoforms of the estrogen receptor, Toxicol. Lett. 121, 167 - 178.

32. Brown, D. W.; Graupner, P. R.; Sainsbury, M.; Shertzer, H.G. (1991) New Antioxidants Incorporating Indole and indoline Chromophores Tetrahedron 1991, 47, 4383 - 4408.

33. Butera, J. A.; Antane, S. A.; Hirth, B.; Lennox, J. R.; Sheldon, J. H.; Norton, N. W.; Warga, D.; Argentieri, T. M.; (2001) Indazolylamino quinazolines and pyridopyrimidines as inhibitors of the EGFr and C-erbB-2 Bioorg. Med. Chem. Lett. 11, 2093 - 2098.

34. Cerri, R.; Boatto, G.; Pau, A.; Sparatore, F.; Cima, L. (1991) Synthesis and Cytotoxicity Studies of Cyclohepta[$[b]$ indoles, Benzo[6,7]cyclohepta[1,2-b]indoles, Indeno[1,2-b]indoles, and Benzo[ $a]$ carbazoles Farmaco Ed. Sci. 46, 369 - 378.

35. Cerri, R.; Boatto, G.; Pau, A.; Sparatore, F.; Manca, P. (1988) Synthesis, antimicrobial and antioxidant activities of some indole analogues containing naphthyridine and pyrimidonaphthyridine systems Farmaco Ed. Sci.43, 113 - 124.

36. Kuehm-Caubere, C.; Caubere, P.; Jamart-Gregoire, B.; Pfeiffer,B.; Guardiola-Lemaitre, B. (1999) Synthesis and reactivity of styrylchromonesEur. J. Med. Chem. Chim. Ther.34, 51 - 62.

37. Joseph, B.; Chapellier, V.; Merour, J.-Y.; Leonce, S. (1998) Synthesis, Cytotoxicity, DNA Interaction and Topoisomerase II Inhibition Properties of Etrahydropyrrole[3,4- $a$ ]carbazole-1,3-dione and Tetrahydropyrido[3,2-b]pyrrole[3,4-g]indole-1,3-dione Heterocycles 48, 1423 -1430.

38. Joseph, B.; Alagille, D.; Merour, J.-Y.; Leonce, S. (2000) 2-Chloro-7,8,9,10-tetrahydrocyclohepta[b]indol6(5H)-one, Chem.Pharm. Bull. 2000, 48, 1872 - 1876.

39. Bor-Cherng H., Yea-Fen J., Yi-Ling C. and Shiow L., (2006) Synthesis and Cytotoxicity Studies of Cyclohepta $[b]$ indoles,Benzo[6,7]cyclohepta[1,2- $b]$ indoles, Indeno[1,2-b]indoles, and Benzo $[a]$ carbazoles, J.Chin. Chem. Soc. 53, No. 3, 647-662. 